\title{
Combined astrometric catalogue EOC-3 ${ }^{\star}$
}

\section{An improved reference frame for long-term Earth rotation studies}

\author{
J. Vondrák and V. Štefka
}

\begin{abstract}
Astronomical Institute, Academy of Sciences of Czech Republic, Boční II, 14131 Prague 4, Czech Republic e-mail: [vondrak; stefka]@ig.cas.cz
\end{abstract}

Received 16 June 2006 / Accepted 13 October 2006

\section{ABSTRACT}

\begin{abstract}
Context. In the past, we collected the astrometric observations of latitude/universal time variations made worldwide at 33 observatories. These observations, re-analyzed with the Hipparcos Catalogue, were then used to determine the Earth Orientation Parameters (EOP) at 5-day intervals, covering the interval 1899.7-1992.0. Later on, new astrometric catalogues (such as ARHIP or TYCHO-2) appeared as combination of Hipparcos/Tycho positions with ground-based catalogues. These catalogues yield more accurate proper motions than the original Hipparcos Catalogue.

Aims. Many of the objects observed in the programmes of monitoring Earth orientation from the ground are double or multiple systems, having non-negligible periodic motions. We aim at obtaining a star catalogue with improved proper motions and quasi-periodic terms reflecting orbital motions of the stars observed in these programmes.

Methods. We used about 4.5 million observations of latitude/universal time variations, and combined them with the catalogues ARIHIP, TYCHO-2, etc. to obtain a new Earth Orientation Catalogue (EOC). These are the same ground-based observations that were used to construct the previous version, EOC-2. Spectral analysis of ground-based data is performed to discover which of the observed objects display periodic motion, and the method of least squares is then used to estimate corresponding amplitudes and phases.

Results. The third version of the catalogue, EOC-3, contains 4418 different objects (i.e., stars, components of double stars, photocenters). Our attempt leads to a description of more accurate paths of the observed objects that will be eventually used for another determination of the Earth Orientation Parameters in the twentieth century.
\end{abstract}

Key words. reference systems - astrometry - catalogs

\section{Introduction}

During the twentieth century, many observatories monitored Earth orientation (polar motion, universal time) in space by optical astrometry. In the past, we collected these observations, covering the interval 1899.7-1992.0 and derived three solutions of the Earth Orientation Parameters (EOP), based on optical observations of latitude/universal time/altitude variations made with 47 instruments at 33 observatories. These solutions were denoted as OA97 (Vondrák et al. 1998), OA99 (Vondrák et al. 2000), and OA00 (Ron \& Vondrák 2001). All these solutions were worked out in the reference frame of the Hipparcos Catalogue (ESA 1997), but about $20 \%$ of positions/proper motions of Hipparcos stars had to be corrected because of the systematic deviations in their positions extrapolated from the Hipparcos epoch backwards. These corrections reflected problems mostly connected with double and multiple star systems.

Later on, new catalogues (such as FK6, GC+HIP, TYC2+HIP, ARIHIP and TYCHO-2) with improved proper motions appeared, as a combination of the Hipparcos and/or Tycho Catalogues with ground based catalogues. The latter two, i.e., ARIHIP (Wielen et al. 2001c) and TYCHO-2 (Høg et al. 2000), form the basis of construction of the new Earth Orientation

* Table 1 is only available in electronic form at the CDS via anonymous ftp to cdsarc.u-strasbg.fr $(130.79 .128 .5)$ or via http://cdsweb.u-strasbg.fr/cgi-bin/qcat?]/A+A/463/783
Catalogue (EOC) whose general ideas were outlined by Vondrák $\&$ Ron (2003). We constructed its first version EOC-1 based on the combination of the observations made only in local meridian with ARIHIP, TYCHO-2, and some other catalogues (Vondrák $\&$ Ron 2006). Then we added the observations by astrolabes, using the method of equal altitudes, and constructed the version EOC-2 (Vondrák 2004), containing 4418 different objects (stars, components of double stars, photocenters). Based on this catalogue we prepared another solution of EOP, called OA04 (Vondrák \& Ron 2005). Since the periodic character of the residuals for certain stars became evident, we decided to construct another version of the catalogue, called EOC-3, and also containing information on the periodicity of some of the observed objects. To this end, we use exactly the same Earth orientation observations as in constructing EOC-2.

\section{Construction of EOC-3}

On average, each star was observed in Earth orientation programmes about thousand times, with the precision of one observation of about 0.2 , usually in a relatively long time interval (decades). It means that the proper motions (and sometimes even positions) can be determined with a precision competing with that of ARIHIP, TYCHO-2, and other similar catalogues. Thus the combination with these input catalogues brings an important improvement. The basis of all subsequent calculations is the preliminary catalogue EOC-0 that contains all objects 
observed in the programs of Earth orientation. Their positions and proper motions are taken over from the ARIHIP (2995 objects), TYCHO-2 (1248 objects), Hipparcos (144 objects), and PPM catalogues (Roeser \& Bastian 1991; Bastian \& Roeser 1993), 28 objects. Only three stars were not found in any of these catalogues, therefore their positions and proper motions were taken from the local catalogue of the instrument in question. In the case of ARIHIP, which contains three different solutions, LTP (long-term prediction) mode is used. In this catalogue, Wielen et al. (2001c) introduced a classification of stars according to their "astrometrical excellency", by assigning certain number of asterisks to each of them. The higher the number of asterisks (from one to three), the more "astrometrically excellent" the star is. We use this information when combining our observations with this and other catalogues (see below).

\subsection{Data used}

We use about 4.5 million observations with 47 instruments of different types, working at 33 observatories. They can be divided into four groups, according to the method of observation that they use:

1. 10 Photographic Zenith Tubes (PZT) that observe near local zenith and determine both latitude and universal time:

2 in Mizusawa, Japan, covering 1959.0-1993.1;

1 in Mount Stromlo, Australia, covering 1957.8-1985.7;

1 in Ondřejov, Czech Republic, covering 1973.1-2002.6;

1 in Punta Indio, Argentina, covering 1971.6-1984.5;

2 in Richmond, Florida, USA, covering 1949.8-1989.4;

3 in Washington DC, USA, covering 1915.8-1992.0.

2. 7 Photoelectric Transit Instruments (PTI), observing the transits of stars over local meridian and determining only universal time:

1 in Irkutsk, Russia, covering 1979.1-1992.0;

1 in Kharkov, Ukraine, covering 1973.0-1992.0;

1 in Nikolaev, Ukraine, covering 1974.4-1992.4;

3 in Pulkovo, Russia, covering 1959.7-1994.0;

1 in Wuhang, China, covering 1981.9-1987.2.

3. 16 visual Zenith Telescopes (ZT) and similar instruments (Visual Zenith Tube - VZT, Floating Zenith Telescope FZT), measuring only latitude:

7 ZT's at ILS stations (Carloforte, Cincinnati, Gaithersburg, Kitab, Mizusawa, Tschardjui, Ukiah), covering 1899.7-1979.0;

1 ZT in Belgrade, Yugoslavia, covering 1949.0-1986.0;

1 ZT in Blagovestschensk, Russia, covering 1959.0-1992.0;

1 ZT in Irkutsk, Russia, covering 1958.2-1991.0;

1 ZT in Józefoslaw, Poland, covering 1961.8-1996.0;

1 FZT in Mizusawa, Japan, covering 1967.0-1984.8;

2 ZT's in Poltava, Ukraine, covering 1949.7-1990.4;

1 ZT in Pulkovo, Russia, covering 1904.7-1995.0;

1 VZT in Tuorla-Turku, Finland, covering 1963.7-1989.1.

4. 14 equal altitude instruments (Danjon astrolabes - AST, photoelectric astrolabes - PAST, and circumzenithals - CZ) that observe a combination of latitude/universal time: 1 PAST in Beijing, China, covering 1979.0-1987.8;

$1 \mathrm{CZ}$ in Bratislava, Slovakia, covering 1987.0-1991.9;

1 PAST in Grasse, France, covering 1983.2-1992.0;

1 AST in Paris, France, covering 1956.5-1983.0;

1 AST in Pecný, Czech Republic, covering 1970.0-1992.0;

1 CZ in Prague, Czech Republic, covering 1980.2-1992.0;

1 AST in Santiago de Chile, covering 1965.9-1990.9;

2 PAST's in Shaanxi, China, covering 1974.0-1992.0;
1 AST + 1 PAST in Shanghai, China, covering 1962.0-1985.0;

1 AST in Simeiz, Ukraine, covering 1977.0-1991.0;

1 AST in Wuhang, China, covering 1964.0-1986.2;

1 PAST in Yunnan, China, covering 1980.7-1991.3.

Generally speaking, latitude observations are used to improve declinations, observations of universal time are used to improve right ascensions, and observations by the method of equal altitude contribute to both coordinates.

In principle, there is no problem with groups 1 and 2 that give the individual corrections of right ascension and declination separately, for each star. A complication arises for instruments in group 3 that always observed star pairs. For these instruments we directly obtain from the observations a mean difference of declinations of two stars, and we face a problem of how to separate them. We chose to create, from each such series of star pairs, two new series (one for each of the two stars) by using the weighting proportional to their squared uncertainties taken from the input catalogue, referred to the center of the interval covered by observations. Group 4 is a special case, since declination and right ascension are mixed in one observation. It is sometimes impossible to derive both coordinates from these observations alone (e.g., if only one of the two possible transits - east or west - is observed). However, if combined with observations by at least one of the other three groups, and/or with virtual observations produced from catalogue entry (see below), both coordinates can be easily resolved.

\subsection{Improving positions and proper motions}

We first found corrections of positions and proper motions, using a strategy very similar to the one used in constructing EOC-2. We determine the positions of the observed stars with respect to astrometrically excellent stars (in the sense of Wielen et al. 2001c), observed by the same instrument. To this end, we used the procedure in following steps:

1. all available data were re-computed into the reference frame of EOC-0, using the new IAU2000A model of precessionnutation (Mathews et al. 2002);

2. the differences of latitude $\delta \varphi$, universal time $\delta \mathrm{UT}$, or altitude $\delta h$ were computed from the mean values of the same night based on only astrometrically excellent stars;

3. the differences for the same star at different epochs were subject to linear regression;

4. the stars with significant deviations were checked for multiplicity, and in positive cases the displacement of the reference point (very often photocenter) from the catalogue entry was estimated. In this case, the catalogue position in EOC-0 was corrected and the procedure above (steps 1-3) was repeated once more. For these stars (but also for many others) the periodic parts were determined later on (see the next subsection);

5. combination of the differences obtained in step 2 with EOC-0 was made. To this end, each entry of the star of catalogue EOC-0 was represented by three virtual observations (both in right ascension and declination) in three epochs covering one century:

$t_{1}=t_{\circ}-90, t_{2}=t_{\circ}, t_{3}=t_{\circ}+10$,

where $t_{\circ}$ is the mean epoch of the catalogue position of the respective star. The epochs $t_{1}, t_{3}$ above were chosen subjectively; however, the method of combination described below 
is quite robust with respect to their choice, and practically any values $t_{1}, t_{3}$ lead to the same results. Since the values of these "observations" should represent their deviations from EOC-0, they were implicitly set to zero. Their uncertainties were calculated from the catalogue standard errors of the position $\sigma_{\circ}$ and proper motion $\sigma_{\mu}$ as

$$
\begin{aligned}
\sigma_{1}^{2} & =9000 \sigma_{\mu}^{2}, \\
\sigma_{2}^{2} & =\sigma_{\circ}^{2} /\left[1-\left(\sigma_{\circ} / \sigma_{\mu}\right)^{2} / 900\right], \\
\sigma_{3}^{2} & =1000 \sigma_{\mu}^{2},
\end{aligned}
$$

which would reproduce the catalogue entry exactly if the virtual observations are subject to linear regression. The sigmas, determined from Eqs. (1) were used to calculate the weights of the three virtual observations $p_{i}=4 \times 10^{4} / \sigma_{i}^{2}$, where $\sigma_{i}$ are given in milliarcseconds (mas); all real observations (deviations calculated above) were assigned weights equal to 1 . This procedure assumes the uncertainty of a ground-based observation of about 200 mas. For the stars whose catalogue entry was taken from Hipparcos we took into consideration the relatively short interval (less than four years) used for determining proper motions, and de-weighted the virtual observations by multiplying $\sigma_{\mu}$ by two. This assured that the weights of virtual observations of Hipparcos stars at distant epochs $t_{1}, t_{3}$ diminished by a factor of four. Then the weighted linear regression through all real and virtual observations was made to yield the combined position/proper motion. To this end, we used the following observation equations for the three types of observations (latitude $\varphi$, universal time UT, altitude $h$ ), respectively:

$$
\begin{aligned}
\delta \varphi= & \Delta \delta+\Delta \mu_{\delta}\left(t-t_{\circ}\right) \\
15.041 \delta \mathrm{UT} \cos \varphi= & \Delta \alpha^{*}+\Delta \mu_{\alpha}^{*}\left(t-t_{\circ}\right) \\
\delta h= & \Delta \alpha^{*} \sin q+\Delta \mu_{\alpha}^{*}\left(t-t_{\circ}\right) \sin q \\
& +\Delta \delta \cos q+\Delta \mu_{\delta}\left(t-t_{\circ}\right) \cos q .
\end{aligned}
$$

Here $\Delta \alpha^{*}, \Delta \mu_{\alpha}^{*}$ stand for $15 \Delta \alpha \cos \delta, 15 \Delta \mu_{\alpha} \cos \delta$, and $q$ is the parallactic angle of the star. The first two parts of Eqs. (2) are used for virtual observations of declination and right ascension with their weights, respectively, and they are mixed with any of the three equations for real observations, according to their type. The method of weighted least squares is used to estimate the corrections $\Delta \alpha^{*}, \Delta \mu_{\alpha}^{*}, \Delta \delta, \Delta \mu_{\delta}$.

The corrections of position and proper motion found from the procedure above were then added to EOC- 0 . The residuals were grouped within each year and from them, the mean annual values of deviations in right ascension and declination calculated. These values were further used, in a subsequent section, to find the periodic motions of observed objects.

Star numbers in the catalogue are assigned as follows:

- Hipparcos number is assigned if the star is contained in the Hipparcos Catalogue and is either single or the same entry as given in original catalogue was observed.

- Hipparcos number +300000 is assigned if the star is contained in the Hipparcos Catalogue, but another component than that given as entry in the original catalogue was observed. In this case, the displacement of the observed component from catalogue entry is estimated (see step 4 of the procedure described above).

- Number greater than 200000 is assigned if the star is not contained in the Hipparcos Catalogue.
Sometimes different components of the same catalogue entry were observed by different instruments. In general, visual instruments are less liable to observe photocenter instead of a component than photographic or photoelectric instruments; the human eye seems to be a better detector of light, able to distinguish close objects (about 1-2" seems to be a limit). In these cases, we keep two different entries in the catalogue, with two different numbers (differing by 300000 ).

\subsection{Finding periodic parts}

The method of spectral analysis proposed by Lomb (1976) appeared to be the best for discovering the periodicity because it is designed for unevenly distributed data with gaps. Namely we used the subroutine in C, provided by Press et al. (1992). From the analysis of the mean annual values of residuals (see above), we typically obtained one or two periods for declination and right ascension for each star, except for the stars for which there were no observations, that were observed too briefly to suppose periodicity, or for which the spectral analysis yielded no significant results.

The periods obtained by Lomb method were accepted if they were longer than 2 years and shorter than the interval covered by the observations. If we found two very near peaks in one coordinate (declination or right ascension), we assumed that they reflect only a single period whose value we calculated as a weighted mean, with the weights depending on their spectral power. The same procedure was applied for slightly different periods found in both coordinates for the same object, so that we use always the same periods for both coordinates.

Although most stars for which periods were found by spectral analysis belonged to the Hipparcos Catalogue, the Sixth Catalog of Orbits of Binary Stars (Hartkopf \& Mason 2006) appeared to be the best for checking for multiplicity because it was also based on Hipparcos and contained all important information. However, we did not use the information on stars with periods shorter than one year because our data were averaged annually. The comparison of the periods found by spectral analysis and those contained in the Sixth Catalog was made more or less intuitively, roughly following a relative 20 per cent tolerance. The number of periods we could use from the Sixth Catalog was only 100 , because others were out of interval $2-100$ years, or the appropriate star was observed too briefly to confirm periodicity. So our method detected only about one half of them. The main reason why we did not detect more periods was the existence of big gaps in data, which caused problems in estimating the sine and cosine terms.

The periods found in the Sixth Catalog had priority over the ones determined by the Lomb method. In $80 \%$ of the cases the differences between our spectral analysis and the Catalog's periods were smaller than about 10 per cent of the period in question. Only in a few cases (e.g., Figs. 3 and 5), when the Catalog's periods were several times longer than the interval covered by our observations or shorter than two years, did the Lomb method yield periods that did not satisfy the condition given by Eq. (4). In these cases we tried to use the Catalog's entry and found it satisfactory. There are three different cases:

1. the period was found by spectral analysis only. There are 535 such cases;

2. the period was found both by spectral analysis and in the Sixth Catalog, their difference was smaller than a $20 \%$ relative tolerance, the Catalog's period was accepted. There are 40 such cases; 

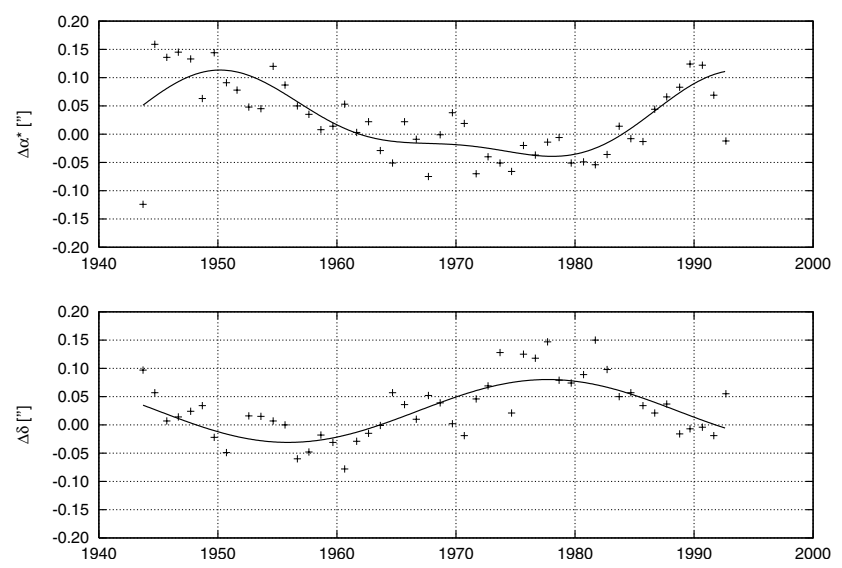

Fig. 1. Star No. 111841 was observed nearly 49 years. The analysis showed one significant period, 43.6 years, in both coordinates.

3. the difference between the two values for the period exceeded the tolerance, the period found by spectral analysis did not satisfy the condition (4), but for the period taken over from the Sixth Catalog the condition was met. There were only 10 such cases.

For each period we estimate a sine and cosine term. To account for elliptic motion, we always also estimate second harmonic terms (i.e., with double frequency). Thus we obtain either four coefficients (in case of one significant period), or eight coefficients (if we found two periods). The corresponding observation equations are:

$$
\begin{aligned}
y_{i}= & A+C \cos 2 \pi f_{1} t_{i}+D \sin 2 \pi f_{1} t_{i} \\
& +C_{1} \cos 4 \pi f_{1} t_{i}+D_{1} \sin 4 \pi f_{1} t_{i} \\
& +E \cos 2 \pi f_{2} t_{i}+F \sin 2 \pi f_{2} t_{i} \\
& +E_{1} \cos 4 \pi f_{2} t_{i}+F_{1} \sin 4 \pi f_{2} t_{i},
\end{aligned}
$$

where $t_{i}$ is time in years elapsed from $\mathbf{J} 2000.0$, frequency $f_{j}=$ $1 / P_{j}$ (for periods $P_{j} \neq 0$ ), and $y_{i}$ is a coordinate (right ascension or declination) referred to $t_{i}$. Solving these equations by the method of least squares, we obtain the unknown coefficients $A$, $C, D, \ldots$ and their accuracies characterized by uncertainties $\sigma_{A}$, $\sigma_{C}, \sigma_{D}, \ldots$ Then we test the coefficients for statistical significance - if the estimated amplitude, calculated from the coefficients $C, D$ (and similarly for $C_{1}, D_{1}, \ldots$ ) as $M=\sqrt{C^{2}+D^{2}}$ does not satisfy the relation

$M>2.5 \sqrt{\frac{C^{2} \sigma_{C}^{2}+D^{2} \sigma_{D}^{2}}{C^{2}+D^{2}}}$,

the corresponding term is neglected and the process of approximation is repeated again, until all remaining coefficients are statistically significant. The coefficient $A$ is added to right ascension or declination. Coefficients $C, D, C_{1}, \ldots$ are amplitudes that in fact define the angular separation and position angle as a function of time. They could eventually serve to determine the orbits, but it is beyond the scope of this paper. Several typical examples of approximating periodic motions are given in Figs. 1 through 5. Each of them shows the observed difference in position (crosses) and the periodic approximation (full line). It is evident that, in the cases displayed, the motion is better described by a curve than by a simple linear regression.

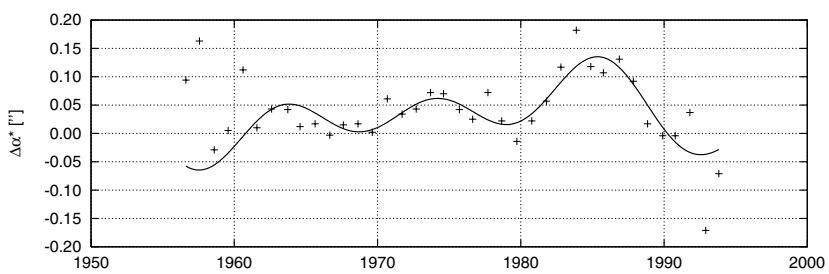

Fig. 2. Star No. 114831 was observed nearly 78 and 38 years in declination and right ascension, respectively. Even though both coordinates were observed, a periodicity was found only in one of them, right ascension. We found two periods, 37.2 and 11.4 years.

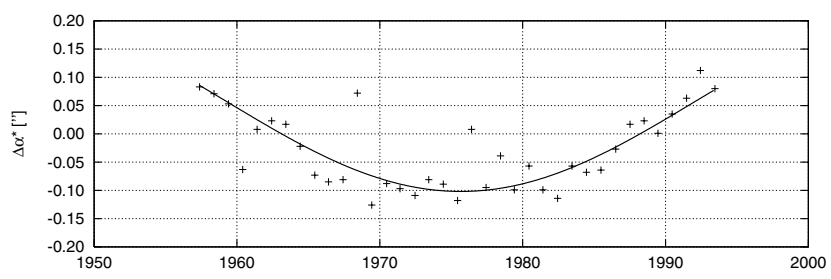

Fig. 3. Star No. 86974 was observed nearly 36 years in both coordinates. The spectral analysis revealed a period, but we found in the Sixth Catalog of Orbits of Visual Binary Stars that it is a double star with a period of 65 years. Although this period is longer than the interval covered by our observations, we used it for both coordinates. Since the periodicity in declination did not prove to be significant (Eq. (4)), we had to neglect it.

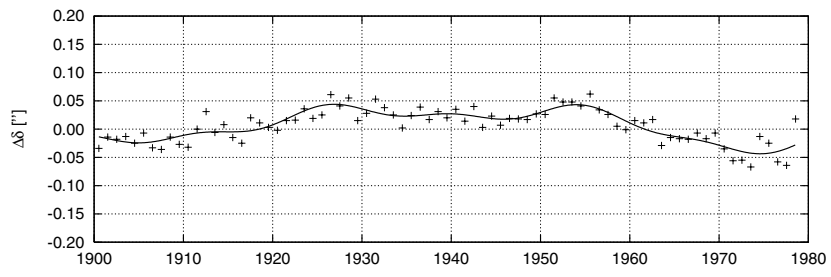

Fig. 4. Star No. 96491 was observed nearly 79 years in declination; Lomb's method revealed two periods, 78.1 and 28.4 years.

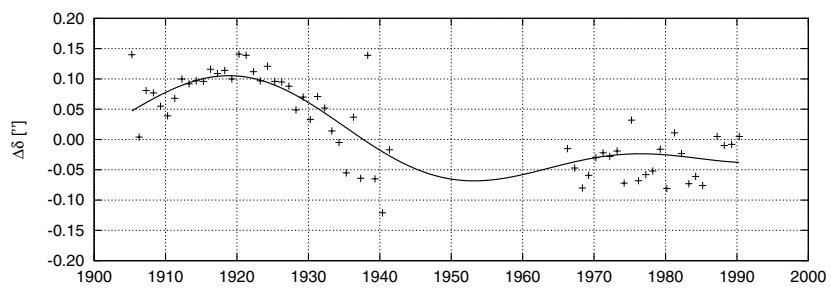

Fig. 5. Star No. 63503 was observed nearly 85 years in declination. Spectral analysis showed one period, but parallel to this we found from the Sixth Catalog of Orbits of Visual Binary Stars that it is a double star with the period of 106.7 years. Although this period is longer than the interval covered by our observations, we accepted its value.

\section{Catalogue description and statistics}

The whole catalogue EOC-3 is included in Table 1, available only in electronic form at CDS. In addition to "classical" information, such as positions, proper motions, their sigmas, parallaxes, radial velocities, and magnitudes, it also contains the information on periods (up to two values), sine/cosine coefficients $C, D, C_{1} \ldots$, and their uncertainty (only one value is given since they are more or less equal for different coefficients), and the information how the period was obtained. A detailed description of the catalogue is given in the Readme file accompanying the catalogue. 


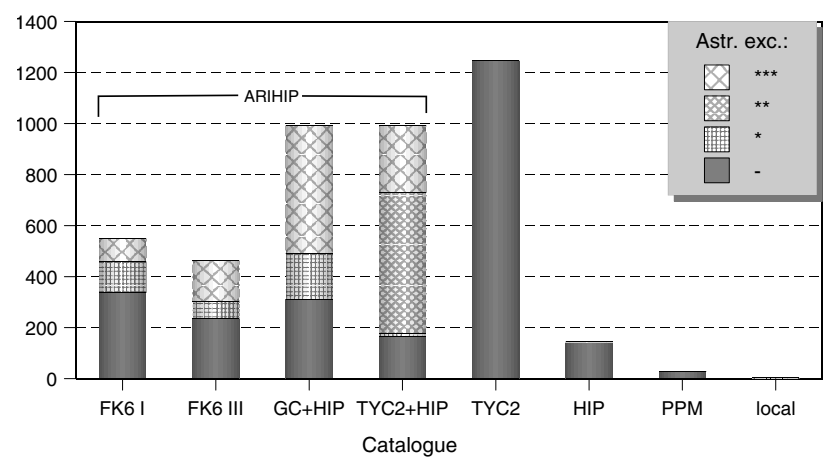

Fig. 6. Number of stars in EOC-3 according to the original catalogues, and their "astrometric excellency" according to Wielen.

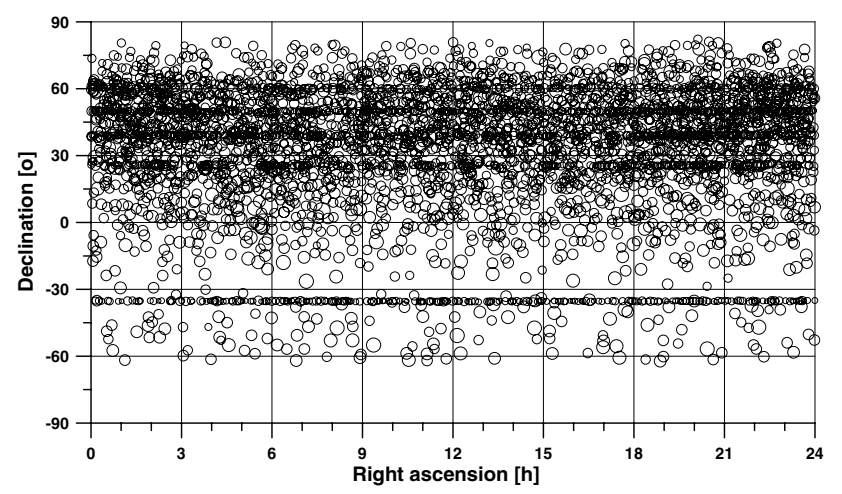

Fig. 7. Distribution of EOC-3 stars on celestial sphere.

The statistics of the catalogues from which the stars in EOC-3 originate is shown in Fig. 6. ARIHIP is in fact a selection of the best stars taken over from several catalogues, combined from ground-based and space-based catalogues:

- FK5 combined with Hipparcos: FK6, Part I (Wielen et al. 1999) and FK6, Part III (Wielen et al. 2000);

- Boss GC combined with Hipparcos: GC+HIP (Wielen et al. 2001a);

- Tycho-2 combined with Hipparcos: TYC2+HIP (Wielen et al. 2001b).

The best stars, in an astrometrical sense, are those with three asterisks. Only $44 \%$ of the stars in EOC-3 (i.e., fewer than two thousand) are classified by Wielen as "astrometrically excellent" (i.e., marked with at least one asterisk). Only these stars were used to define the reference frame in which our catalogue is given. Many of the stars with no asterisk in the ARIHIP group, shown in dark gray in the figure, are double or multiple systems. Most of the objects in the catalogue are contained in the Hipparcos Catalogue - only 85 of them are not. Out of these, 58 objects are other components than those directly listed in Hipparcos, and 585 have significant periodic motions.

The distribution of EOC-3 stars on the celestial sphere is depicted in Fig. 7. The asymmetric distribution with respect to the equator is evident. It is caused by the distribution of the observatories on the globe; only three of them are located on southern hemisphere. There are also visible several horizontal lines along which the star distribution is condensed - these correspond to PZT's that observe stars in only narrow declination bands. The distribution of visual magnitudes of the stars contained in the catalogue is displayed in Fig. 8. Great majority of them $(68 \%)$ are in the range from magnitude 5 to 8 , which is given by the observation technique used (mostly visual instruments). The

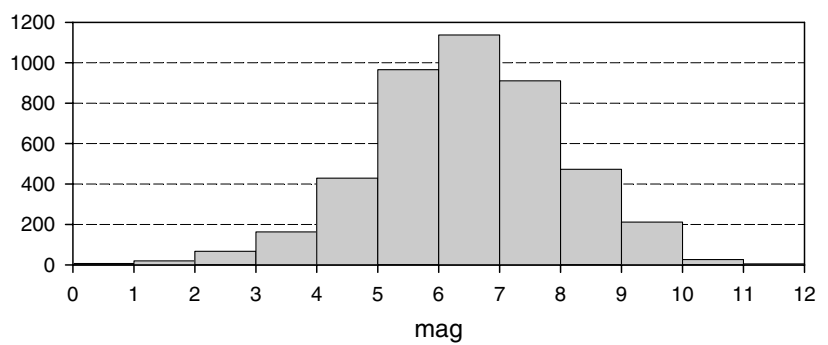

Fig. 8. Magnitude distribution of stars in EOC-3.

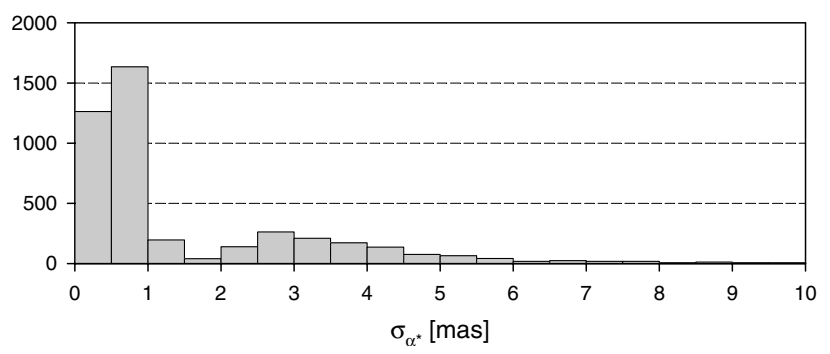

Fig. 9. Distribution of standard errors of right ascension* in EOC-3.

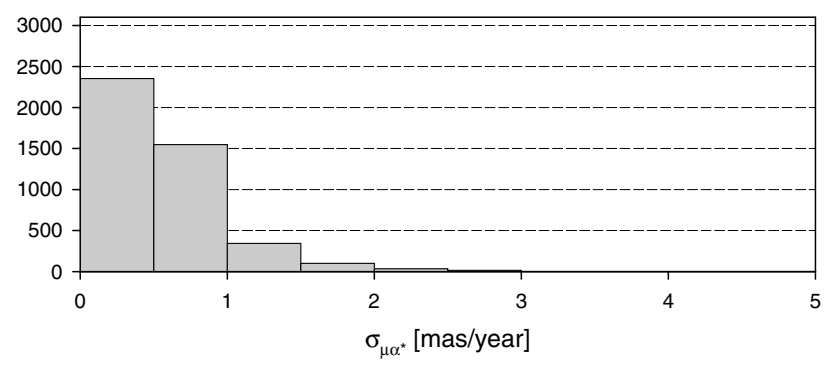

Fig. 10. Distribution of standard errors of proper motion in right ascension* in EOC-3.

distribution of standard errors in positions and proper motions of the new catalogue is depicted in Figs. 9 through 12, both for right ascension and declination. All histograms displayed there use bins 0.5 mas wide. Two remarkably distinct maxima (a bigger one between 0 and 1 and a smaller one around 3 mas) in Figs. 9 and 11 (standard errors in right ascension and declination) reflect the fact that most of our stars originate from two different populations (in a statistical sense) with different accuracy - Hipparcos and Tycho-2. However, these features are completely missing in Figs. 10 and 12 that display standard errors in proper motion in right ascension and declination, respectively. It is caused by the combination of the input catalogues with ground-based observations with a much longer history that improves proper motions, especially those in declination.

The formal standard errors of positions and proper motions are practically identical to those of EOC-2; only a very small improvement (by several per cent) can be seen for the stars with estimated periodic parts where the residuals of ground-based observations diminished. This can be ascribed to the fact that we used almost the same method to determine the linear part of EOC-3 as in constructing EOC-2 - the standard errors are mainly dictated by ARIHIP and TYCHO-2 with dominant weights, and only to a lesser extent by the accuracy of ground-based observations. The median values of uncertainties of the amplitudes of periodic terms are 10.6 and 8.6 mas for right ascension and declination, respectively. 


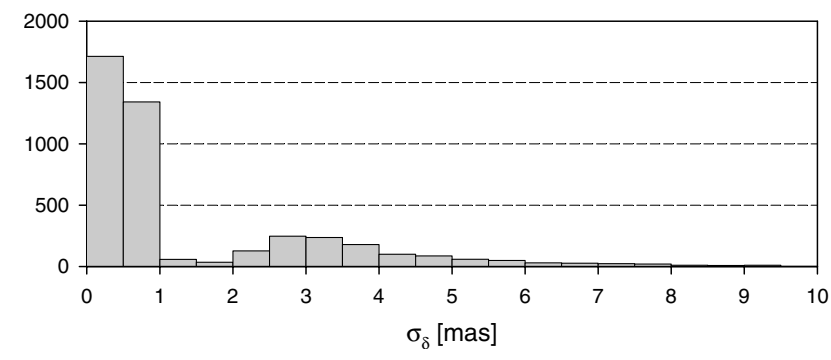

Fig. 11. Distribution of standard errors of declination in EOC-3.

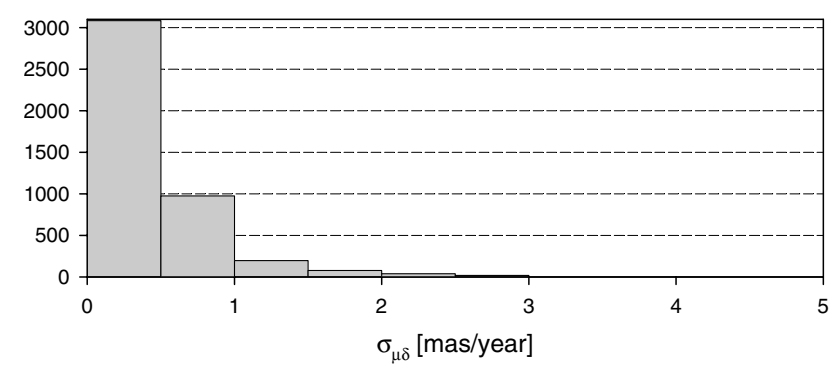

Fig. 12. Distribution of standard errors of proper motion in declination in EOC-3.

\section{Conclusions}

The new Earth Orientation Catalogue EOC-3 is constructed to be more accurate than the preceding versions, EOC- 1 and EOC-2. The catalogue is "tailored" for the purpose of being used for long-term Earth rotation study. Namely we suppose that the non-linear representation of the proper motion by a linear plus periodic term should yield a better approximation for calculating apparent positions of the stars. The new catalogue EOC-3 is planned to be used in a newly prepared solution of the Earth Orientation Parameters from optical astrometry in the last century.

Acknowledgements. This study was made possible thanks to grant No. LC506 Recent Geodynamics, awarded by the Ministry of Education, Youth, and Sports of the Czech Republic. The authors would also like to acknowledge the comments of an anonymous referee who helped us to improve both the text and the catalogue itself.

\section{References}

Bastian, U., \& Roeser, S. 1993, Positions and Proper Motions - South, Astron. Rechen-Inst., Heidelberg

ESA 1997, The Hipparcos and Tycho Catalogues, ESA SP-1200

Hartkopf, W. I., \& Mason, B. D. 2006, The Sixth Catalog of Orbits of Visual Binary Stars, US Naval Observatory

Høg, E., Fabricius, C., Makarov, V. V., et al. 2000, A\&A, 355, L27

Lomb, N. R. 1976, Ap\&SS, 39, 447

Mathews, P. M., Herring, T. A., \& Buffet, B. A. 2002, J. Geophys. Res., 107

Press, W. H., Teukolsky, S. A., Vetterling, W. T., \& Flannery, B. P. 1992, Numerical Recipes in C, The Art of Scientific Computing, 2nd edn. (Cambridge Univ. Press)

Roeser, S., \& Bastian, U. 1991, Positions and Proper Motions - North, Astron. Rechen-Inst., Heidelberg

Ron, C., \& Vondrák, J. 2001, in Journées 2000 Systèmes de référence spatiotemporels, ed. N. Capitaine (Observatoire de Paris), 201

Vondrák, J. 2004, Serb. Astron. J., 168, 1

Vondrák, J., \& Ron, C. 2003, in Journées 2002 Systèmes de référence spatiotemporels, ed. N. Capitaine, \& M. Stavinschi (Observatoire de Paris), 49

Vondrák, J., \& Ron, C. 2005, Kinematics and physics of celestial bodies, Suppl. No. 5, 305

Vondrák, J., \& Ron, C. 2006, in Proc. IAU XXV Joint Discussion 16, The ICRS Maintenance and Future Realizations, ed. R. Gaume, D. McCarthy, \& J. Souchay (US Naval Observatory), 112

Vondrák, J., Pešek, I., Ron, C., \& Čepek, A. 1998, Publ. Astron. Inst. Acad. Sci. Czech R., 87, 1

Vondrák, J., Ron, C., \& Pešek, I. 2000, in Polar motion: Historical and Scientific Problemes, ed. S. Dick, D. D. McCarthy, \& B. Luzum, Proc. IAU Coll., 178, ASP Conf. Ser., 208, 206

Wielen, R., Schwan, H., Dettbarn, C., et al. 1999, Veröff. Astron. Rechen-Inst. Heidelberg, 35 (Karlsruhe: Kommissions-Verlag G. Braun)

Wielen, R., Schwan, H., Dettbarn, C., et al. 2000, Veröff. Astron. Rechen-Inst. Heidelberg, 37 (Karlsruhe: Kommissions-Verlag G. Braun)

Wielen, R., Schwan, H., Dettbarn, C., et al. 2001a, Veröff. Astron. Rechen-Inst. Heidelberg, 38 (Karlsruhe: Kommissions-Verlag G. Braun)

Wielen, R., Schwan, H., Dettbarn, C., et al. 2001b, Veröff. Astron. Rechen-Inst. Heidelberg, 39 (Karlsruhe: Kommissions-Verlag G. Braun)

Wielen, R., Schwan, H., Dettbarn, C., et al. 2001c, Veröff. Astron. Rechen-Inst. Heidelberg, 40 (Karlsruhe: Kommissions-Verlag G. Braun) 